\title{
EL BOLETÍN DE EDUCACIÓN PARAGUAYA EN LOS AÑOS I95O Y LAS PRESCRIPCIONES PARA LOS PROFESORES
}

\section{The Boletin de Educación Paraguaya in the igsos and prescriptions for teachers}

Cinthya Lorena LARREA VIERA

Universidade Federal da Grande Dourados

Correo-e: cinthylo@hotmail.com

Kênia Hilda Moreira

Universidade Federal da Grande Dourados

Correo-e: keniamoreira@ufgd.edu.br

Recibido: 6 de septiembre de 2020

Envío a informantes: 12 de septiembre de 2020

Aceptación definitiva: 22 de noviembre de 2020

Resumen: Tratamos de identificar y analizar los contenidos prescritos en la primera fase del Boletín de Educación Paraguaya - Revista Mensual de Orientación e Información Pedagógica, entre 1956 y 1959, bajo el gobierno dictatorial de Alfredo Stroessner. Partimos de la hipótesis de que este impreso estimuló nuevas representaciones alrededor del quehacer docente en Paraguay, a la luz de los preceptos de la escuela activa, teniendo a los docentes como lectores visados. Cuestionamos las prescripciones para establecer nuevos patrones de comportamiento y actitudes para los profesores paraguayos al analizar los primeros 37 números de la revista. Como referenciales teórico-metodológicos de análisis nos basamos en la historia cultural y en las bibliografías sobre historia de la educación paraguaya. Concluimos que los estímulos divulgados en el Boletín para producir nuevas representaciones sobre la figura del profesor, en el Paraguay en la década de 1950, lo ubican como protagonista de la Reforma Educativa Paraguaya de 1957, inculcándoles un modelo de conducta ante la sociedad, una autoridad moral y saberes científicos modernos y patrióticos.

Palabras Clave: prensa pedagógica; formación docente; historia de la educación en el Paraguay. 
AвSTRACT: We aim to identify and analyze the contents prescribed in the first phase of the Boletin de Educación Paraguaya - Monthly Journal of Guidance and Pedagogical Information, between 1956 and 1959, under the dictatorial government of Alfredo Stroessner. We work on the hypothesis that the periodical newsletter stimulated new representations around the teaching work in Paraguay, in light of the active school precepts, having teachers as target readers. We wondered about the prescriptions that wished to establish new patterns of behaviors and attitudes to Paraguayan teachers after analyzing the first 37 issues of the newsletter. As theoretical-methodological references for analysis, the text is based on cultural history and the bibliographies on the history of Paraguayan education. We conclude that the stimuli spread in the periodical to produce new representations about the role of the teacher, in Paraguay in the I950s, places him/ her as main character of the Paraguayan Educational Reform of 1957, instilling them as a role model for society, a moral authority, an example of patriotic and filled with modern and scientific knowledge.

KEY WORDS: pedagogical press; teacher training; History of Education in Paraguay.

\section{Introducción}

$\mathrm{E}$

STE ARTÍ́CULO SE PROPONE IDENTIFICAR Y ANALIZAR los contenidos prescritos en el Boletín de Educación Paraguaya (BEP) - Revista Mensual de Orientación e Información Pedagógica, sobre el quehacer docente en Paraguay, entre 1956 y 1959, período de la Reforma Educativa de $1957^{1}$. Nuestra hipótesis es que el Boletín, al tener a los docentes como lectores visados ${ }^{2}$, buscaba estimular nuevas sensibilidades alrededor del quehacer docente en Paraguay, a la luz de los preceptos de la escuela activa, en conformidad con la Reforma. Dicho Boletín fue creado, editado y dirigido por Emilio Uzcátegui ${ }^{3}$, quien estuvo en Paraguay, desde 1955 hasta 1959, prestando asesoramiento a la Reforma, en nombre de la Unesco ${ }^{4}$. La fundación y difusión de este impreso, por lo tanto, forma parte del proyecto de reforma educativa en Paraguay con mediación de la Unesco.

Al llegar al país, Uzcátegui realiza una exploración de los diarios y revistas en circulación en Paraguay en aquel entonces ${ }^{5}$ y concluye que «no existe en el país nin-

La Reforma Educativa paraguaya de 1957 abarcó diferentes etapas de la enseñanza y se denominó de esta manera porque lleva en consideración el Decreto n. 29.594, de 27 de septiembre de 1957, por el cual fue aprobado el Plan de Actividades Educativas para el ciclo de preescolar y para las escuelas primarias, como afirma QuinTANA DE HORAK (I995). El desarrollo de la reforma transcurrió de modo desigual en los diferentes niveles educativos, pues fueron reformulados gradualmente y los decretos que los convalidaban fueron expedidos en diferentes años.

2 GALVÃo y Melo (2019) explican ese concepto al declarar que se trata del lector previsto por el autor y/o editor en el momento de la producción del objeto de lectura.

Para mayores informaciones sobre Emilio Uzcátegui, jefe de la misión educativa en Paraguay, recomendamos el artículo «Perspectiva Educativa y política de Emilio Uzcátegui y su prospectiva desde la educación», de Ruth Tatiana Fonseca Morales y Edgar Martínez Arco (20I8).

Organización de las Naciones Unidas para la Educación, la Ciencia y la Cultura.

Al hacer la exploración de los medios de comunicación existentes en Paraguay en la década de 1950, Uzcátegui destaca la existencia de tres diarios: La Tribuna, El País y La Patria. Los dos últimos, clasificados por él como «voceros de la Junta de Gobierno del Partido Colorado, todos ellos son modernos y cuentan con buena información especialmente extranjera. Su tirada en conjunto asciende a 32000 ejemplares diarios» (Boletín..., 1957, n. ${ }^{\circ}$ 9-10, p. 96). En relación a las revistas existentes, clasificadas por él como culturales, menciona: Alcor, La Estrella, Revista de APA, Revista del Centro Médico, Paraguay Industrial 
guna revista ni publicación de carácter educativo y los diarios, por su muy reducido espacio, no pueden dar lugar a la inserción de artículos indispensables para preparar el ambiente de la reforma» (Uzcátegui, Informe I, 1955, p. 7).

[...] juzgo, por esto, la imperiosa necesidad que la Misión edite mensual o bimestralmente algún boletín, revista o publicación periódica en general destinada a repartirse gratuitamente entre los profesores y las personas interesadas en la reforma educativa, para que así se conozca, explique y sedimente el trabajo de la reforma. (Uzcátegui, Informe I, $1955, \mathrm{p} \cdot 7)^{6}$

Uzcátegui consideró de extrema necesidad un periódico pedagógico que pudiera «orientar, explicar y cimentar el proceso de la reforma en curso» (Uzcátegui, Informe 6, 1956, p. 8), creando, así, el Boletín de Educación Paraguaya - Revista Mensual de Orientación e Información Pedagógica.

El primer número de la revista fue publicado en setiembre de 1956, como medio de divulgación y comunicación de la reforma y misión de la Unesco, que estaban en curso. El periódico pedagógico circuló hasta 1971, presentando, según Viera (2018), dos fases: la primera, de 1956 a 1959, con 37 números localizados y la segunda, de 1963 a $197 \mathrm{I}^{7}$, con 3I números localizados. En la primera fase la producción y circulación mensual y gratuita del Boletín fue organizada y editada por la Unesco y el Ministerio de Educación y Culto (MEC) se responsabilizó de la impresión. En la segunda fase, la revista fue producida bajo la responsabilidad del MEC con el apoyo técnico de la Unesco ${ }^{8}$. En este artículo, nos centramos en la primera fase del Boletín.

Destacamos que la reforma educativa aquí analizada, así como la producción y circulación del Boletín como una «Revista Mensual de Orientación e Información Pedagógica», transcurrieron durante la dictadura civil-militar en Paraguay, bajo el comando del general Alfredo Stroessner, quien fue presidente del país entre 1954 a 1989 . El Ministerio de Educación y Culto fue creado por Stroessner por el Decreto-Ley n. 46/1954, transfiriendo el Departamento de Culto, que pertenecía al Ministerio de Relaciones Exteriores, al Ministerio de la Educación, según Quintana de Horak (1995, p. I24). Como justificación el decreto expone «que la atención al funcionamiento de numerosas instituciones educacionales pertenecientes a entidades religiosas, diseminadas por toda la República, corresponde por su carácter específico al Ministerio de Educación, lo que recomienda la transferencia del Departamento de Culto de la Cancillería al Ministerio de Educación» (Decreto-Ley n. 46 del 8 de noviembre de 1954 apud Velázquez Seiferheld y D’Alessandro, 2017, p. 63).

y Comercial y algunas revistas especializadas, publicadas por la Facultad de Derecho y Ciencias Sociales y por el Ministerio de Justicia y Trabajo.

6 Emilio Uzcátegui, educador ecuatoriano, que estuvo a cargo de la misión de la Unesco en Paraguay, escribió informes regulares reportando la evolución de su trabajo al frente de la reforma educativa. El primer informe fechado el is de diciembre de 1955 y el último el 9 de noviembre de 1959 , totalizando 23 a lo largo de sus cuatro años de misión.

Existe un lapso de tiempo en la publicación del Boletín entre 1960 y 1963, esa brecha de tiempo coincide con la conclusión de la primera misión de la Unesco en Paraguay. Los indicios sugieren que con la partida del director Emilio Uzcátegui la publicación haya sido suspendida en ese período.

8 Para identificar las dos fases del Boletín se tuvieron en consideración las diferencias presentadas en sus elementos materiales: diseño, diagramación, tapas, colores, número de imágenes, periodicidad. Sumándose a esto, tenemos la forma de obtención del Boletín por el público al que era destinado. Cabe destacar que en la primera fase su distribución al magisterio se daba de forma gratuita y en la segunda pasa a ser cobrado. 
El Ministro de Hacienda, general César Barrientos, es presentado como financiador del Boletín en su primera fase. La ausencia de anuncios y propagandas que ayudarían en el apoyo presupuestario del impreso indican que su existencia dependía de la financiación de ese ministerio en colaboración con la Unesco. La revista, como se anuncia, mantuvo una periodicidad mensual. El siguiente cuadro sintetiza informaciones materiales de las ediciones de la primera fase, analizadas en este artículo, teniendo en consideración que un texto no existe fuera de los objetos escritos, como afirma Chartier (1990):

CuAdro i. Síntesis de las ediciones que componen la primera fase del Boletín

\begin{tabular}{|c|c|c|c|c|c|c|c|}
\hline Año & $\begin{array}{c}\text { N. } \\
\text { publicados }\end{array}$ & $\begin{array}{l}\text { Páginas } \\
\text { por n. }\end{array}$ & $\begin{array}{c}\text { Formato } \\
(\mathrm{cm})\end{array}$ & Artículos & Colores & $\begin{array}{l}\text { Número de } \\
\text { fotografías }\end{array}$ & $\begin{array}{l}\text { Número de } \\
\text { Ilustraciones }\end{array}$ \\
\hline 1956 & 4 & 46 & $26,5 \times 18$ & $5 \mathrm{I}$ & 2 & 6 & - \\
\hline 1957 & $\mathrm{I} 2$ & 45 & $26,5 \times 18$ & $\mathrm{I} 22$ & 2 & 5 & 2 \\
\hline 1958 & I2 & 44 & $26,5 x$ I8 & 95 & 2 & 2 & 32 \\
\hline 1959 & 9 & $4 \mathrm{I}$ & $26,5 \times 18$ & $3 \mathrm{I}$ & 2 & - & - \\
\hline
\end{tabular}

Fuente: Elaborado por las autoras, en conformidad al modelo presentado por Biccas (2008).

El periódico no indica el tiraje, pero el director resalta que «su distribución es gratuita y se hace preferentemente entre el magisterio primario y secundario» (Boletín..., I959, n..$^{\circ} 36$, p. 2I).

De acuerdo a las estadísticas oficiales, en 1957, la población total del Paraguay era alrededor de 1.700.000 personas en todo el territorio (Sánchez, 1957). El número de escuelas primarias en 1957 era de 2.040 , con 283.853 alumnos inscritos atendidos por 9.927 profesores. Había 36 escuelas normales, 26 del Estado y io particulares, con 834 alumnos y 3.920 alumnas. En lo tocante a la educación secundaria, en 1959 funcionaron I03 colegios, 30 públicos y 73 particulares, con un total de I.IO4 profesores (Uzcátegui, 1959). Los datos mencionados ayudan a proyectar una estimativa acerca de la cantidad de instituciones que podrían haber recibido el Boletín en ese período, considerando que su público eran los docentes formados y en formación de enseñanza primaria y secundaria. De este modo, se suman alrededor de 2.I79 escuelas y colegios con aproximadamente II.3I2 profesores. Por lo tanto, si la mitad de esas instituciones hubiesen recibido el Boletín tendríamos más de I.ooo ejemplares por número. Al tratar de la difusión y prestigio de la educación paraguaya a través de la revista al exterior, Uzcátegui afirmó en 1959 que «son cerca de 400 ejemplares que circulan en el exterior, predominantemente en América Latina» (Boletín..., 1959, n. ${ }^{\circ}$ 36, p. 2I). Con esto, es posible inferir que la circulación interna sería muy superior a ese número.

Para analizar los contenidos del Boletín de Educación Paraguaya como prensa pedagógica", en la segunda mitad de la década de 1950, conscientes de que «un texto no existe sino porque hay un lector para otorgarle significación» (Chartier, 1993, p.

9 Utilizamos prensa pedagógica según Hernández Díaz (20I8). 
24), y buscando indicios de prescripciones sobre la práctica docente que indiquen nuevas representaciones ${ }^{10}$ sobre los profesores, dividimos este artículo en tres partes: Prescripciones de lectura para los profesores en el Boletín; Prescripciones de método para el quehacer de los profesores en el Boletín; y Prescripciones de cómo se tiene que ver a la figura del profesor según el Boletín.

\section{Prescripciones de lectura para los profesores en el Boletín}

Creemos que las motivaciones para mantener la periodicidad del Boletín suponen una oportunidad de educar los sentidos de sus lectores. El propio proceso de recepción mensual de un impreso hecho para ellos instaura un nuevo hábito de lectura. Una de las estrategias de Uzcátegui como director de la revista fue el de presentar al Boletín de Educación Paraguaya como el vocero de la reforma y guía para los docentes, legitimándolo como impreso pedagógico oficial del Gobierno, por lo tanto, digno de confianza. La ausencia de otros periódicos educacionales oficiales contribuyó a la concretización de esa estrategia ${ }^{\text {II }}$. En su primer informe y en el Boletín, reiteradas veces Uzcátegui expone la necesidad de construir un vehículo de informaciones que se aproximara a sus lectores.

En la portada de la revista su eslogan ya anunciaba la necesidad de orientar e informar a los educadores sobre los cambios que se avecinaban a raíz de la reforma educativa en transcurso. En la primera fase del impreso fueron publicados 244 artículos cuyos temas trataban de actividades pedagógicas, planes de estudios y formación pedagógica dirigidas a una práctica dentro de la metodología de la Escuela Activa ${ }^{12}$ y sobre normativas para la conducta docente.

En esta primera fase del Boletín identificamos ocho secciones, agrupadas en dos categorías: el primer agrupamiento corresponde a las secciones con obras de lectura sugeridas para los maestros y el segundo se relaciona con los mecanismos adoptados para la divulgación de la reforma en Paraguay, las acciones de Unesco e informaciones sobre el proprio Boletín.

Para Umberto Eco, el texto prevé una cooperación del lector para que el mensaje sea alcanzado, actualizado. Él explica que «generar un texto significa aplicar una estrategia que incluye las previsiones de los movimientos del otro» (Eco, I993, p. 79). Por lo tanto, se hace indispensable que durante el análisis de la revista se considere el lector modelo previsto por los autores y el editor ${ }^{13}$. En la argumentación propues-

io El concepto de representación que usamos está en conformidad con la definición de CHARTIER (1996).

II Mencionamos el concepto de estrategia en la perspectiva de CERTEAU (I998).

12 La reforma educacional de I922/1924, conocida como Reforma Cardociana, fue considerada como una de las más elementales para el avance de la educación en Paraguay. Por ser una reforma que abarcó varios niveles educativos y por presentar la propuesta de la Escuela Nueva, lo que se refiere en especial a la formación de profesores.

I3 Utilizamos el termino editor en singular porque existen indicaciones, en los informes hechos por Uzcátegui y en los artículos del Boletín, que él ha sido si no el único, el principal responsable de la edición del Boletín. Incluso, en su último informe fechado el 9 de noviembre de 1959, informa que había dejado preparados los ejemplares n. ${ }^{\circ} 37$ y 38 listos para publicación, aunque él ya no estuviera presente. Coincidentemente, después del término de su misión en Paraguay la publicación del Boletín queda interrumpida hasta 1963. 
ta por Eco (1993), el autor de un texto supone que el lector modelo debe poseer un conjunto de competencias que posibiliten moverse interpretativamente al paso que el autor se mueve generativamente. No obstante, Eco atribuye un mayor alcance al autor por medio del texto, no limitándose a prever competencias, sino instituirlas. «De manera que prever el correspondiente Lector Modelo no significa sólo 'esperar' que éste exista, sino también mover el texto para construirlo» (Eco, I993, p. 8I). Por consiguiente, a la luz de este concepto intentamos identificar aquellos elementos que fueron construyendo al lector visado en las páginas del Boletín.

Hechas esas consideraciones, emprendemos al análisis de los artículos publicados en las páginas del Boletín de Educación Paraguaya, escritos por diferentes autores paraguayos y colaboradores de la Unesco. Pero, en su mayoría, los textos con indicaciones de lecturas fueron escritos por Emilio Uzcátegui, el cual se consagró como el autor más frecuente al escribir 88 textos, de un total de 244 artículos publicados en el Boletín en su primera fase. Esto corresponde al $36 \%$ de la producción total incluida en el periódico. Los temas abarcados en el Boletín no presentaban una línea editorial específica. A pesar de esto, fueron organizados con el objetivo de orientar a los lectores hacia una formación pedagógica moderna. Examinamos las obras propuestas por el periódico, expuestas en el cuadro que sigue, a fin de identificar aquellas cuyos temas prescribieron actitudes y prácticas para una nueva sensibilidad del quehacer y ser profesor en Paraguay:

Cuadro 2. Secciones identificadas en el Boletín de Educación Paraguaya

\begin{tabular}{|l|c|}
\hline \multicolumn{1}{|c|}{ Nombre de sección } & Números del BEP \\
\hline Libros útiles & I; 6; II \\
\hline Notas bibliográficas & I4, I8 y 2I \\
\hline Bibliografía & $24 ; 28 ; 34-35$ \\
\hline Páginas literarias & 27 \\
\hline Valiosas opiniones sobre BEP, palabras de estímulo & 4 \\
\hline La reforma de la educación paraguaya juzgada en el exterior & $7 ; 8 ; \mathrm{II} ; \mathrm{I} 3 ; \mathrm{I} 4 ; \mathrm{I} 6 ; \mathrm{I} 7$ \\
\hline En torno a la reforma educativa en Paraguay & $\mathrm{I} 8$ \\
\hline Palabras de estímulo & $2 ; 22$ \\
\hline
\end{tabular}

Fuente: Elaborado por las autoras en base al Boletín de Educación Paraguaya.

En las secciones Libros útiles, Bibliografías, Notas bibliográficas y Páginas literarias fueron presentados pequeños artículos con sugerencias de lectura, reseña crítica de libros, especialmente para los profesores y alumnos de las escuelas normales. Al analizar las obras encargadas en el Boletín, presentadas en el Cuadro 3, identificamos libros didácticos/compendios destinados a los alumnos de secundaria en general y también a los profesores ya formados o en formación: 
EL BOLETÍN DE EDUCACIÓN PARAGUAYA EN LOS AÑOS I950

Y LAS PRESCRIPCIONES PARA LOS PROFESORES

CINTHYA LORENA LARREA VIERA Y KÊNIA HILDA MOREIRA

Cuadro 3. Obras sugeridas en las secciones del Boletín de Educación Paraguaya

\begin{tabular}{|c|c|c|}
\hline $\begin{array}{l}\text { N. }{ }^{\circ} B E P \\
\text { (Año) }\end{array}$ & Títulos sugeridos en el $B E P$ & $\begin{array}{l}\text { Autores de las } \\
\text { obras sugeridas }\end{array}$ \\
\hline I $\quad(1956)$ & Introducción a la literatura & Manuel E. Arguello \\
\hline (1957) & $\begin{array}{l}\text { Cómo reconocer el estilo y el autor de una } \\
\text { obra musical } \\
\text { Dianas de Sol (poesías para niños) }\end{array}$ & $\begin{array}{l}\text { Juan Max Boettner } \\
\text { Lorenzo F. D’Auria }\end{array}$ \\
\hline (1957) & $\begin{array}{l}\text { Ciencias Naturales/Estadística educacional } \\
\text { de ross } \\
\text { La educación del pueblo }\end{array}$ & $\begin{array}{l}\text { Ministerio } \\
\text { de Educación } \\
\text { Luis Reissing }\end{array}$ \\
\hline I4 (1957) & $\begin{array}{l}\text { La enseñanza de la lengua materna en la es- } \\
\text { cuela secundaria }\end{array}$ & Fidel C. González \\
\hline$(1958)$ & Era tecnológica y la educación & Luis Reissing \\
\hline$(1958)$ & $\begin{array}{l}\text { Antología de los grandes maestros; Ética pro- } \\
\text { fesional; Métodos Educativos; Régimen Le- } \\
\text { gal de la Educación Secundaria; Filosofía de } \\
\text { la Educación }\end{array}$ & $\begin{array}{l}\text { Jorge Castro } \\
\text { Harrison }\end{array}$ \\
\hline$(1958)$ & El arte en la educación & Emilio Uzcátegui \\
\hline$(1958)$ & $\begin{array}{l}\text { Compendio de Historia Paraguaya } \\
\text { El arte en la educación }\end{array}$ & $\begin{array}{l}\text { Julio César Chavez } \\
\text { Emilio Uzcátegui }\end{array}$ \\
\hline $34-35 \quad$ (1959) & $\begin{array}{l}\text { Compendio de Historia Americana } \\
\text { Las primeras civilizaciones: Oriente y Grecia } \\
\text { Panorama de la Educación Paraguaya (am- } \\
\text { pliada y corregida) } \\
\text { El arte en la educación } \\
\text { Disertaciones Filosóficas }\end{array}$ & $\begin{array}{l}\text { Julio César Chávez } \\
\text { Luis G. Benítez y } \\
\text { Óscar Paciello } \\
\text { Emilio Uzcátegui } \\
\text { Emilio Uzcátegui } \\
\text { Juan Vicente Ra- } \\
\text { mírez }\end{array}$ \\
\hline
\end{tabular}

Fuente: Elaborado por las autoras en base al Boletín de Educación Paraguaya.

Entre las prescripciones de lectura para los profesores en el Boletín, fueron identificadas obras con contenidos para materias distintas (literatura, ciencias, historia del Paraguay, historia de América) y obras destinadas a la formación del profesor (métodos educativos, bases legales de la secundaria, filosofía de la educación, educación y tecnología). Entre las recomendaciones de lecturas se percibe un énfasis para «el arte en la educación», con la inclusión de la literatura y las obras musicales, además de «poesías para niños». Intuimos que el arte fuera una manera de proponer prácticas activas en el aula, en conformidad con el método intuitivo.

Las recomendaciones de lectura para los maestros y profesores en el cuadro anterior también evidencian un énfasis en las obras publicadas por el propio editor del Boletín. Entre otras obras de educación, Uzcátegui fue autor de El arte en la Educa- 
ción (1957) y Páginas de Cultura y Educación (1953), buscando estimular la práctica de actividades artísticas en el aula.

Las recomendaciones de lectura en el Boletín trataron de construir en los profesores una sensibilidad de nuevas actitudes en las cuales a un buen profesor le debería gustar leer, instruirse, y habilitarse en la teoría y práctica de la pedagogía moderna. Como señala Eco (1993) al tratar del lector modelo, el Boletín de Educación Paraguaya incluyó el texto impreso en sus páginas con el objetivo de construir el profesor ideal, en conformidad a la Reforma.

En adelante presentamos de modo más detallado los métodos que deberían ser utilizados por los profesores, según el Boletín, según la Reforma.

\section{Prescripciones de método para el quehacer de los profesores en el Boletín}

En su primer número, el Boletín define la educación activa como una educación que «se produce solamente por la acción, por la acción interesada, nacida de necesidades que sienten los educandos. Esto es la enseñanza activa, en donde los alumnos no se limitan a escuchar, sino toman parte activa en el aprendizaje» (Boletín..., 1956, n. ${ }^{\circ}$ I, p. 28). La propuesta pedagógica difundida por el Boletín incentivó la participación más activa del alumno. Consecuentemente, fueron hechas muchas críticas por integrantes de las comisiones de la reforma al currículo anterior, clasificándolo como enciclopédico y facilitador de un aprendizaje memorístico, fragmentado, opuesto a todas las verdaderas finalidades educativas. Al respecto, el periódico buscó inculcar una nueva sensibilidad en el docente y también en el alumno, confiriéndoles practicas más participativas.

Para alcanzar la cientificidad en la educación, el rol del profesor fue señalado como crucial. En ese sentido, el impreso prescribe la formación adecuada de los profesores de la siguiente forma: «Los maestros de la escuela elemental necesitan orientar a los alumnos en la aplicación de la verdad científica» (Boletín..., 1956, n. ${ }^{\circ}$ 2, p. 22). Las nuevas orientaciones pedagógicas fortalecen la adquisición de conocimientos por experimentación y por comprobación científica. Sin embargo, ese objetivo solo sería alcanzado a través de una mejora en la formación de los profesores. De esta manera, el Boletín indica que «[...] los maestros para el logro de todo esto, necesitan una adecuada base de conocimientos científicos, tener una perspectiva correcta de ciencia, estar familiarizado con el método científico y además conocer las comunidades en que se desenvuelven los alumnos [...]» (ibídem, p. 22). A ese respecto, se suman las creaciones de los departamentos e institutos creados con esa finalidad: Departamento de Investigaciones Psicopedagógicas, Centro de Documentación Pedagógica y Producción de Material Didáctico y el Instituto de Física y Química, todos contaron con el asesoramiento de la Unesco.

La creación de dichos departamentos tenía por objetivo poner en practica la reforma que empezaba a tomar forma en los planes de estudios y programas, cuyo mayor objetivo era la modernización de la formación docente. En el discurso inaugural del Instituto de Física y Química, el Boletín publicó el siguiente mensaje: «La formación del profesorado es tan importante como un buen plan ya que no es lo mismo conocer una materia que enseñarla. Como bien lo dice un autor enseñar es un hacer y que por 
lo tanto requiere un arte de ese que hacer» (Boletín..., $1957, \mathrm{n} .^{\circ} \mathrm{I} 6, \mathrm{p} .30$, subrayado original).

Podemos afirmar que el Boletín sirvió de vehículo para la diseminación de ideales pedagógicos que orientaban a los docentes sobre los diversos teóricos, entre los cuales se destacan Decroly, Dewey y Cláparede. Se percibe que al prescribir esos saberes y reglar esas actitudes, el periódico paulatinamente instruye al docente para la asimilación de métodos de enseñanza y de las nuevas prácticas educativas. Seleccionamos algunos de los títulos cuyas páginas presentan indicaciones para la nueva educación, moderna y activa: La escuela y la comunidad; Actividades recreativas; Pensamiento y obra de un gran educador paraguayo; Escuela Primaria, Activa y Funcional; Percepción y Educación; Charlas sobre metodología de la segunda enseñanza; Ovidio Decroly; Diferentes clases de planes de estudio; Planes y programas y el desarrollo del niño; La Educación Fundamental es una enseñanza activa; entre otros. A continuación, presentamos los artículos publicados en el Boletín, donde identificamos reflexiones sobre nuevas propuestas metodológicas a los docentes:

Cuadro 4. Artículos pedagógicos publicados en el Boletín (1956-1959)

\begin{tabular}{|c|c|c|}
\hline \multicolumn{2}{|c|}{ Año $\left(\right.$ n. $\left.^{\circ}\right)$} & Artículos pedagógicos publicados en el Boletín \\
\hline 1956 & $\left(\right.$ (n. ${ }^{\circ}$ I) & $\begin{array}{l}\text { La escuela y la comunidad; Actividades recreativas; El Programa de Lengua- } \\
\text { je en la escuela primaria }\end{array}$ \\
\hline 1956 & $\left(\mathrm{n}^{\circ}{ }_{2}\right)$ & La enseñanza del Idioma nacional; Ciencias \\
\hline 1956 & $\left(\right.$ n. $\left.^{\circ} 3\right)$ & Pensamiento y obra de un gran educador paraguayo \\
\hline 1956 & $\left(n^{\circ} 4\right)$ & $\begin{array}{l}\text { Escuela Primaria, Activa y Funcional; El Estudio de la Historia y su valor } \\
\text { educativo en la formación de la juventud }\end{array}$ \\
\hline 1957 & $\left(n .^{\circ} 5\right)$ & $\begin{array}{l}\text { La cooperación de la Estadística en la visión de nuestros problemas educa- } \\
\text { cionales; Áreas de Interés en el aula }\end{array}$ \\
\hline 1957 & $\left(\right.$ n. $\left.{ }^{\circ} 6\right)$ & Percepción y Educación; Charlas sobre metodología de la segunda enseñanza \\
\hline I957 & $\left(\mathrm{n}^{\circ} 7\right)$ & $\begin{array}{l}\text { La educación de adultos. Educación para vivir mejor; El microfilm y sus } \\
\text { aplicaciones a la enseñanza }\end{array}$ \\
\hline 1957 & $\left(\right.$ n. $\left.{ }^{\circ} 8\right)$ & La Música en la Educación Secundaria; Enseñanza de la lectura y la escritura \\
\hline 1957 & $\left(\right.$ (n. $\left.{ }^{\circ} 9-\mathrm{IO}\right)$ & Panorama de la educación en el Paraguay \\
\hline I957 & $\left(\mathrm{n}^{\circ} \mathrm{II}\right)$ & $\begin{array}{l}\text { Panorama de la educación en el Paraguay; La Iniciación Estética por discos } \\
\text { en las Escuelas de Párvulos; Pedagogía y Didáctica en la Enseñanza Agrícola } \\
\text { Elemental; Modalidades y tendencias actuales de la enseñanza de los párvu- } \\
\text { los en las Escuelas Primarias del Reino Unido }\end{array}$ \\
\hline I957 & $\left(\mathrm{n}^{\mathrm{o}} \mathrm{I2}\right)$ & $\begin{array}{l}\text { Desarrollo de la Comunidad; Los Maestros Bolivianos. Orientaciones di- } \\
\text { dácticas para la segunda enseñanza. LA PATRIA (28/03-30/o6); Enseñanza } \\
\text { Actual de las Matemáticas en la educación media }\end{array}$ \\
\hline 1957 & $\left(\mathrm{n}^{\circ} 3\right)$ & $\begin{array}{l}\text { Educación de adultos en zonas rurales; La educación de los niños en comu- } \\
\text { nidades dispersas o aisladas; Ovidio Decroly }\end{array}$ \\
\hline
\end{tabular}




\begin{tabular}{|c|c|c|}
\hline 1957 & $\left(\mathrm{n}^{\mathrm{o}} \mathrm{I} 5\right)$ & Trabajos de Artesanía en las escuelas \\
\hline 1957 & $\left(\right.$ n. $\left.^{\circ} \mathrm{I} 6\right)$ & $\begin{array}{l}\text { Un experimento canadiense sobre formación de Inspectores y Administra- } \\
\text { dores; La Inspección Escolar en el Uruguay; El variado papel del Inspector }\end{array}$ \\
\hline 1958 & $\left(\right.$ n. $^{\circ}$ I 7$)$ & $\begin{array}{l}\text { Obstáculos que los maestros encuentran para la lectura de textos profesiona- } \\
\text { les; Literatura de carácter educativo. Su disponibilidad y acceso a la misma; } \\
\text { Definición y finalidad de la lectura de carácter profesional; El problema de } \\
\text { las lecturas } \\
\text { profesionales de los educadores; Publicaciones profesionales; Obstáculos } \\
\text { para la lectura de carácter profesional }\end{array}$ \\
\hline 1958 & (n. ${ }^{\circ}$ I 8$)$ & $\begin{array}{l}\text { Características sociales y culturales del Paraguay y sus consecuencias en re- } \\
\text { lación con la educación gratuita y obligatoria; La biblioteca pública piloto } \\
\text { de Medellín }\end{array}$ \\
\hline 1958 & $\left(\right.$ n. $\left.^{\circ} 19-20\right)$ & $\begin{array}{l}\text { Los Museos en el Paraguay; Relato Informativo de la I Mesa Redonda sobre } \\
\text { Museos en el Paraguay; Contribución de los Museos a la Educación; Los } \\
\text { Museos como auxiliares educativos; Consideraciones generales sobre los } \\
\text { Museos; Casi la mitad de los adultos del mundo no pueden leer }\end{array}$ \\
\hline 1958 & $\left(\right.$ n. $\left.^{\circ} 2 \mathrm{I}\right)$ & Influencia de la Educación en la Agricultura \\
\hline 1958 & $\left(\right.$ n. $\left.^{\circ} 24\right)$ & Centro de Currículum del SCIDE: Manual del Maestro paraguayo \\
\hline 1958 & $\left(\mathrm{n}^{\circ} 26\right)$ & $\begin{array}{l}\text { Educación Comparada como instrumento de planeamiento; } \\
\text { La conservación y la protección de libros, revistas y periódicos en las regio- } \\
\text { nes tropicales; Impresiones sobre un curso de perfeccionamiento }\end{array}$ \\
\hline 1959 & $\left(\right.$ ( $\left.^{\circ} 27\right)$ & $\begin{array}{l}\text { La Educación Fundamental es una enseñanza activa; Enseñando a estudiar a } \\
\text { los alumnos }\end{array}$ \\
\hline 1959 & $\left(\mathrm{n}^{\circ} 28\right)$ & $\begin{array}{l}\text { Una nueva concepción del dibujo en los colegios y sus resultados en los del } \\
\text { Paraguay; Control de Alimentos }\end{array}$ \\
\hline 1959 & $\left(\mathrm{n}^{\circ} 3 \mathrm{I}\right)$ & $\begin{array}{l}\text { Curso Publicado en Bloominton, Indiana: PHI Delta Kappan, vol. XXXIX, } \\
\text { n. } 6 \text {, marzo de I958. CIE de la Unesco } \\
\text { El factor de las relaciones humanas: estudio del curso en cuanto grupo social; } \\
\text { Las discusiones en grupo y su importancia en la formación de maestros; } \\
\text { Desarrollo de los grupos y educación común a diversos grupos sociales en } \\
\text { la educación de párvulos; Empleo del método de grupo; La educación en } \\
\text { Francia }\end{array}$ \\
\hline 1959 & $\left(\mathrm{n}^{\circ} 32\right)$ & $\begin{array}{l}\text { La educación en la era atómica; Planeamiento de la educación; América } \\
\text { necesita educación; Fundamentos y función de las Unidades de Trabajo y } \\
\text { técnicas de su elaboración y desarrollo; Valoración del arte moderno; Que } \\
\text { significa «arte abstracto» }\end{array}$ \\
\hline
\end{tabular}

Fuente: Elaborado por las autoras en base al Boletín de Educación Paraguaya.

Al seleccionar los artículos, tratamos de identificar en ellos los nuevos sentidos y sensibilidades que se proponían a los profesores. 
Apuntamos la atención sobre uno de ellos: «Ovidio Decroly». Fue uno de los pocos artículos publicados por el Boletín como resultado de esa nueva educación de los sentidos del profesor paraguayo dirigido hacia una educación activa. El texto escrito por las alumnas del séptimo semestre del curso de profesorado de la Escuela Normal n. ${ }^{\circ} 2^{14}$ trata sobre la vida del Dr. Ovidio Decroly, publicado en conmemoración del cincuentenario de la escuela de l'Ermitage. Ese indicio nos permite inferir que los preceptos diseminados por el Boletín estimulaban una reproducción del material leído, incorporándose en la práctica de los alumnos de las escuelas normales. No hemos encontrado en el Boletín otros artículos elaborados por alumnos. Por otro lado, en el n. ${ }^{\circ}$ 36, Uzcátegui afirma que los artículos vehiculados en el periódico sirvieron de fuente para la producción de monografías de los alumnos de las escuelas normales.

Como se ha señalado, los temas de los artículos seleccionados discutían la formación del profesor; recomendaban lecturas; estipulaban metodologías impregnadas a la luz de la pedagogía activa con publicaciones de cursos enteros; enseñaban el cuidado para la preservación de materiales, e indicaban instrucciones sobre áreas específicas del conocimiento (ciencias y arte). Además de eso, instruían sobre cómo enseñar a los alumnos a estudiar y cómo organizar una sala de clase. Había también artículos que retrataban la educación en otros países. Resumiendo: el periódico sirvió de vehículo para la construcción de conductas y valores que deberían ser cultivados por los docentes en las escuelas normales y aquellos ya formados.

\section{Prescripciones de cómo se tiene que ver a la figura del profesor en el Boletín}

El periódico buscó educar la sensibilidad de los profesores para que comprendieran el trabajo como un legado para la nación, más allá de la ganancia financiera, construyendo, así, una nueva sensibilidad sobre el rol docente. En ese sentido, considerar la realidad de los sueldos bajos como insuficientes para ese trabajo se constituía un comportamiento inadecuado. Ese pensamiento era permitido para otros oficios, pero no para el maestro. Él debería considerar como parte de su pago la seguridad y alegría de proporcionar conocimientos.

Sin embargo, las palabras de la profesora paraguaya Joaquina Filizzola ${ }^{15}$ no reflejaban esa aceptación:

El quid en la cuestión para tener buenos maestros ha de consistir siempre en que se les asigne buenos sueldos. El maestro debe ser el obrero o empleado mejor rentado, por ejemplo, ahora, el maestro rural más apartado, debe ganar por lo menos tres mil guara-

${ }^{14}$ Las escuelas normales públicas fueron enumeradas acorde a las progresivas instalaciones en $\mathrm{Pa}$ raguay. La primera escuela normal pública fue denominada Escuela normal n. ${ }^{\circ}$ I "Presidente Franco», instalada en la capital del país (I856). Las escuelas normales $n .^{\circ} 2 \mathrm{y} \mathrm{n.}^{\circ} 3$ poseían dos sucursales, una en la capital y otra en ciudades en el interior, todas las demás eran únicas en su numeración. O sea, no existía en el país otra escuela n. ${ }^{\circ}$ I6, solamente la escuela normal de Pedro Juan Caballero. Las escuelas también podían adoptar un nombre después de la identificación numérica, como es el caso, por ejemplo, de la escuela n. ${ }^{\circ}$ I. Por otro lado, las escuelas normales privadas solamente utilizaban nombres. (Datos encontrados en el informe hecho por el consultor Amando Sacristán para la Unesco en 1967).

is Su nombre aparece una única vez como autora del artículo «Sobre Reforma Educacional», con fecha de setiembre de 1956, en la ciudad de Encarnación. Su texto fue publicado casi un año después de ser escrito y ella no formaba parte de ninguna comisión de reforma. 
níes mensuales por sección, y con subsidio progresivo para los maestros que ejercen en los lugares más alejados de los centros urbanos o de cultura como se hace en México, Argentina, Uruguay, etc. (Boletín..., I957, n. ${ }^{\circ}$ II, 1957).

Esas palabras son indicativas de cierta resistencia e inconformidad con la realidad que los profesores paraguayos vivían. Al hacer la comparación con la situación de otros profesores en América del Sur, percibimos que ella utiliza esa información como crítica a la situación de los profesores paraguayos.

Poco espacio fue otorgado a los profesores y alumnos lectores para que pudiesen expresar sus dudas u opiniones en las páginas del periódico o, si lo hicieron, eso no fue publicado con frecuencia. Reconocemos qué educadores paraguayos contribuyeron a la escritura de los artículos referentes a las reformas y algunos artículos pedagógicos, pero, en su mayoría, esos profesores eran representantes oficiales del Gobierno por formar parte de las comisiones de las reformas educativas o figuras públicas y consideradas peritas en educación. En ese sentido, cuestionamos la ausencia de un espacio para la exposición de la opinión del lector ya que este era estimulado a escribir a la dirección del periódico.

En contrapartida, el Boletín reservó amplio lugar para la divulgación de opiniones de los lectores del Boletín clasificados como «autoridades en educación». En su mayoría, esos lectores eran extranjeros y algunos pocos paraguayos. Las secciones del Boletín Palabras de estímulo, Valiosas opiniones sobre "Boletín de Educación Paraguaya", La reforma de la educación paraguaya juzgada en el exterior y En torno a la reforma educativa en Paraguay divulgaban los mensajes enviados por los lectores ilustres al editor. Los contenidos de los mensajes versaron, principalmente, sobre dos temas: la reforma educativa en el Paraguay y el propio periódico.

\section{Consideraciones finales}

Teniendo a los maestros y profesores de enseñanza secundaria como lectores visados, los textos del Boletín de Educación Paraguaya - Revista Mensual de Orientación e Información Pedagógica, en los años 1950, prescribieron lecturas, métodos, no limitándose con prever competencias, sino instituirlas, como sugiere Umberto Eco. En consonancia a los objetivos de la Reforma Educativa paraguaya, teniendo a la escuela activa como modelo pedagógico, los textos del Boletín intentaron presentar los conocimientos científicos necesarios para la formación docente, además de incentivar la participación activa de los alumnos en el contexto escolar, motivando a los profesores. Los textos en la revista estipularon las metodologías impregnadas a la luz de la pedagogía activa con publicaciones de cursos enteros; enseñaron el cuidado para la preservación de materiales, e indicaron instrucciones sobre áreas específicas del conocimiento prescribiendo sobre el quehacer docente, como prácticas y actitudes que deberían ser cultivadas por los docentes en su practica profesional rutinaria. Además, Emilio Uzcátegui, y los demás autores del Boletín propusieron una mirada hacia al ser docente como un ser influyente, cuyo trabajo estaba directamente relacionado con el éxito o el fracaso del desarrollo de la sociedad paraguaya. En síntesis, los estímulos divulgados en el Boletín, para producir nuevas representaciones sobre la figura del profesor, lo ubican como protagonista de la Reforma, inculcándoles un modelo 
EL BOLETÍN DE EDUCACIÓN PARAGUAYA EN LOS AÑOS I950

Y LAS PRESCRIPCIONES PARA LOS PROFESORES

CINTHYA LORENA LARREA VIERA Y KÊNIA HILDA MOREIRA

de conducta ante la sociedad, una autoridad moral y saberes científicos modernos y patrióticos.

Una cuestión que queda por investigar es la apropiación de estas prescripciones por parte de los profesores paraguayos. Y en esto, considerar la disputa ideológica entre los objetivos del Gobierno dictador con la Reforma y, de otra parte, los objetivos de la Unesco y Emilio Uzcátegui.

\section{Fuentes}

Boletín de Educación Paraguaya - Revista Mensual de Orientación e Información Pedagógica. (1956-1959). Asunción-PY: Ministerio de Educación y Culto (37 números).

Unesco. (I955). Asunción, I5 de diciembre de 1955, pp. I-9. Disponible en: https://unesdoc. unesco.org/ark:/48223/pfoooor8o772?posInSet=8\&queryId=N-EXPLORE-a8312er6-2e8e-4 932-8cia-4c2boaa6i657. Acceso I2 ago. 2020.

Uzcátegui, E. (1953). Páginas de Cultura y Educación. Universidad central. Unesco.

Uzcátegui, E. (1955). Informe I. Misión de Asistencia Técnica en el Paraguay. Unesco. Asunción, diciembre is de 1955. Disponible en: https:/unesdoc.unesco.org/ark:/48223/ pfoooor8o772 ? posInSet=8\&queryId=N-EXPLORE-a8312er6-2e8e-4932-8cia-4c2boaa6i657. Acceso I2 ago. 2020.

Uzcátegui, E. (1956). Informe 6. Misión de Asistencia Técnica en el Paraguay. Unesco. Asunción, 30 sep. 1956. Disponible en: https://unesdoc.unesco.org/ark:/48223/pfoooor80772?posInSet=8\&queryId=N-EXPLORE-a8312er6-2e8e-4932-8cia-4c2boaa61657. Acceso i2 ago. 2020.

Uzcátegui, E. (1957). El arte en la Educación. Librería Ferrero Editorial. Unesco.

Uzcátegui, E. (1959). Panorama de la educación paraguaya. Asunción: Imprenta Nacional.

\section{Bibliografía}

Biccas, M. de S. (2008). O impresso como estratégia de formação: Revista do Ensino de Minas Gerais (1925-1940). Belo Horizonte: Argvmentvm.

Certeau, M. de. (1998). A Invenção do Cotidiano. Petrópolis: Vozes.

Chartier, R. (1990). La historia cultural redefinida: prácticas, representaciones, apropiaciones. Revista Punto de Vista, 39, (Año XIII, diciembre). Buenos Aires.

Chartier, R. (1993). Libros, lecturas y lectores en la Edad Media. Madrid: Alianza.

ChARTIER, R. (1996). El mundo como representación. Estudios sobre historia cultural. Barcelona: Gedisa.

Eco, U. (1993). Lector in fabula. Barcelona: Lumen.

Elias, R. (20I4). Análisis de la reforma educativa en Paraguay: discursos, prácticas y resultados. Buenos Aires: Clacso.

Fonseca, R. T. M. y Arco, E. M. (2018). Perspectiva Educativa y política de Emilio Uzcátegui y su prospectiva desde la educación.

Galvão, A. M. de O. y Melo, J. F. de. (2019). Análise de impressos e seus leitores: uma proposta teórica e metodológica para pesquisas em história da educação. En C. G. VEIGA y M. A. TABORDA (coords.), Historiografia da educação: abordagens teóricas e metodológicas / organizadores Análise de impressos e seus leitores: uma proposta teórica e metodológica para pesquisas em história da educação. . ${ }^{a}$ ed. Belo Horizonte, [MG]: Fino Traço.

Hernández Díaz, J. M.a (ed.). (2018). La prensa pedagógica de los profesores. Salamanca: Ediciones Universidad de Salamanca. 
Quintana DE Horak, C. (1995). La educación escolar en el Paraguay. Apuntes para una historia. Asunción: CEPAgSumANDo-Fundación en Alianza.

Sánchez, S. (1957). Estadística Educacional del año I955 (pp. 16-29). Ministerio de Educación y Culto. SCIDE-Asunción.

Velásquez Seiferheld, D. D’Alessandro, S. (20I7). Relaciones entre autoritarismo y educación en el Paraguay 1869-2012. Un análisis histórico, vol. 3. I954-1989. Asunción-Paraguay: Edición Serpaj.

Viera, C. L. L. (2018). O periódico Boletín de Educación Paraguaya (1956-1959): Escolas normais e formação de professores. Dissertação (Mestrado em Educação). Faculdade de Educação, Universidade Federal da Grande Dourados. 